\title{
Modelling and experimental analysis of the angular distribution of the emitted light from the edge of luminescent solar concentrators
}

Thomas S. Parel, Christos Pistolas, Lefteris Danos*\| and Tomas Markvart

Solar Energy Laboratory, Faculty of Engineering and the Environment, University of Southampton, Southampton, SO17 1BJ, UK

*Email:1.danos@1ancaster.ac.uk, Tel:+44 (0) 1524592860.

" Present address: Department of Chemistry, Lancaster University, Lancaster, LA1 4YB, UK

\begin{abstract}
Luminescent solar concentrators (LSC) have the potential to provide cheap solar electricity by significantly reducing the solar cell area. However, these devices are still at the research level and several aspects of their behaviour need investigation in order to improve efficiencies. Understanding how light is absorbed/emitted and concentrated to the edge of LSCs is required to design a high efficiency device as well as identifying and overcoming the various losses present. One strategy for investigating the photon absorption and transport in LSCs as well as pinpointing the sources of losses in these devices is to look at the luminescence escaping the LSC as a function of angle. This paper presents a new model that reveals the main features of the angular distribution of light escaping a LSC edge. We compare this model with experimental measurements and provide an assessment of non-ideal losses and identify which emission angles are affected most by these losses. We investigated experimentally the effects of the absorption profile of the chromophores and re-absorption on the photon flux travelling at different angles. The effect of back surface reflectors, commonly
\end{abstract}


used to 'recycle lost photons', on the edge emission of LSCs has also been investigated in this work.

Keywords: luminescent solar collectors, angle resolved emission, re-absorption, solar cells 


\section{Introduction}

Luminescent solar concentrators (LSC) were proposed more than thirty-five years ago [1-3] as a concept that could provide low cost solar electricity. In LSCs a host material such as poly-methyl methacrylate (PMMA), or glass is used with luminescent dyes [4-6], inorganic absorbers [7-8] or quantum dots [9-11] either embedded within the bulk material or deposited on the external surface of the substrate as a thin film. The chromophores (organic, inorganic, quantum dot) absorb incident light (direct and diffuse) over a large front surface area and emit at a longer wavelength in the form of luminescence. A large portion of the emitted light is then trapped in the LSC by total internal reflection (TIR) and is guided to the edge of the LSC where attached solar cells can convert the incident light to electricity. The LSC has the ability to concentrate the incident sunlight because of the large area difference between the front face of the LSC and the edge areas and offers advantages over geometric concentrators due to the ability to concentrate diffuse light at much lower fabrication costs.

The first stage of research of this technology started during the late 70's [2,3,12-14]. At the time of writing this paper, the highest published efficiency of LSCs was reported using four GaAs solar cells at the edges of the collector with a $7.1 \%$ solar power conversion efficiency [15]. It has been shown [16], however, that LSCs have the potential to reach efficiencies similar to the Shockley-Queisser (S-Q) limit [17], in particular, it has been shown that LSCs coupled to silicon solar cells could achieve a combined theoretical efficiency limit of $26.8 \%$ [16]. Despite their potential for generating low-cost solar power and entering the PV market, the principal challenge facing LSCs is associated with overcoming fundamental losses.

This observed disparity between experimental and theoretical efficiencies is due to losses that can be broadly classified in two categories, dye related losses and light transport losses. Dye related losses include a limitation in the absorption bandwidth of individual dyes, non-unity 
luminescence quantum yields, and stability issues over extended periods of sunlight exposure. Light transport losses are related with the transport of photons to the edge of the LSC and include re-absorption [18], which results from the partial overlap of the absorption and emission bands, and escape cone losses, which are unavoidable in a TIR arrangement. This loss is exacerbated if the luminescence quantum yield is less than unity.

To increase the optical efficiency of LSCs, mirrors and white scattering layers have been applied at the back of the substrate in order to reduce light lost from the bottom of the LSC [19-21]. When applied with an air gap ensuring TIR is not disrupted and it can increase the absorption length of light.

One technique used to analyze absorption and photon transport in LSCs is to make angular measurements of the luminescence emission escaping the edge of LSCs [22-26]. In [22] the emitted luminescence intensity was measured as a function of detection angle for a LSC consisting of a thin film luminescent film deposited on glass. In later works a cylindrical lens coupled to the LSC edge [23-25] was used to 'see' inside uniformly doped LSC polymer plates. Monte Carlo simulations of the photon flux exiting the LSC edge were also presented in these publications. In [26] thin film and bulk doped LSCs were studied. The edges were not coupled to a lens and a simple numerical model that qualitatively explains fringe-like patterns detected for spot illumination was presented.

To our knowledge, the effect of back surface specular mirrors and white scatterers on the angular dependence of the edge emission in LSCs has not been previously investigated. This study is important since these structures have the potential to increase the efficiency of LSC systems without introducing new losses and will certainly need to be incorporated in any commercial LSC systems. Therefore an understanding of their effect on the optical performance of LSCs is vital. We also present a new model that describes the luminescence emission exiting the LSC edge as a function of angle. Angular resolved measurements of 
bulk doped LSCs are also be presented. The theoretical model will be shown to be able to reproduce the main characteristics of the angularly resolved measurements and can possibly be used in the future as a tool to identify the exact source of non-ideal losses in LSCs. In addition, an examination of the spectrum emitted at different angles has been carried out in order to experimentally illustrate the effect of re-absorption of rays with different path length within the collector.

\section{Theory}

In this section we shall develop expressions describing the luminescence emission exiting a LSC edge (the LSC is assumed to not be attached to any device i.e. mirrors, solar cells etc.)

The first generation (i.e. re-absorption free) luminescence exiting the LSC, $\phi_{\text {out }, 1}$, is directly proportional to :

$$
\phi_{\text {out }, 1}(\theta) \propto \xi(\theta)\left\{1-\Gamma_{1}(\theta)\right\},(1)
$$

where $\theta$ is the zenith angle of detection, $\xi(\theta)$ is an angular loss factor which quantifies nonideal losses (i.e. losses other than due to re-absorption or non-unity luminescence quantum yield) and is assumed to depend on the angle of emission (and therefore the path length photons have travelled). $\Gamma_{1}$ is the angular re-absorption probability for light emitted with the first generation luminescence emission spectrum. It represents the probability that light emitted at an angle $\theta$ will be re-absorbed.

Assuming the azimuthal angle of emission detected is close to $90^{\circ}, \Gamma_{1}$ is given by

$$
1-\Gamma_{1}(\theta)=\int_{\lambda_{1}}^{\lambda_{2}} \frac{\int_{0}^{L} A_{1}(z) e^{\frac{-\alpha_{\mathrm{em}}(\lambda)(L-y)}{\sin \theta}} d y}{\int_{0}^{L} A_{1}(z) d y} f_{1}(\lambda) d \lambda,(2)
$$


where $z$ is the axis measuring the depth of the LSC, $y$ is the axis along the length of the LSC, $A_{1}$ is the emission distribution of the LSC with depth for the first generation luminescence and $\lambda_{1}$ and $\lambda_{2}$ are the lower wavelength limit of absorption and the upper wavelength limit of emission respectively. This is described by the Beer-Lambert law and is defined by the absorption coefficient at the excitation wavelength $\alpha_{\mathrm{ex}} . \alpha_{\mathrm{em}}$ is the absorption coefficient at the emission wavelength, $L$ is the length of the $\mathrm{LSC}, f_{1}$ is the first generation luminescence of the dye without re-absorption, normalised to a unit total emission probability, $\int f_{1}(\lambda) d \lambda=1$ i.e. it is the distribution of the re-absorption free luminescence and $\lambda$ is the emission wavelength.

For different positions within the LSC along the $y$ axis, the emission that reaches the detector at a particular zenith angle $\theta$ (Fig. 1), corresponds to emission originating at a specific depth $z$.

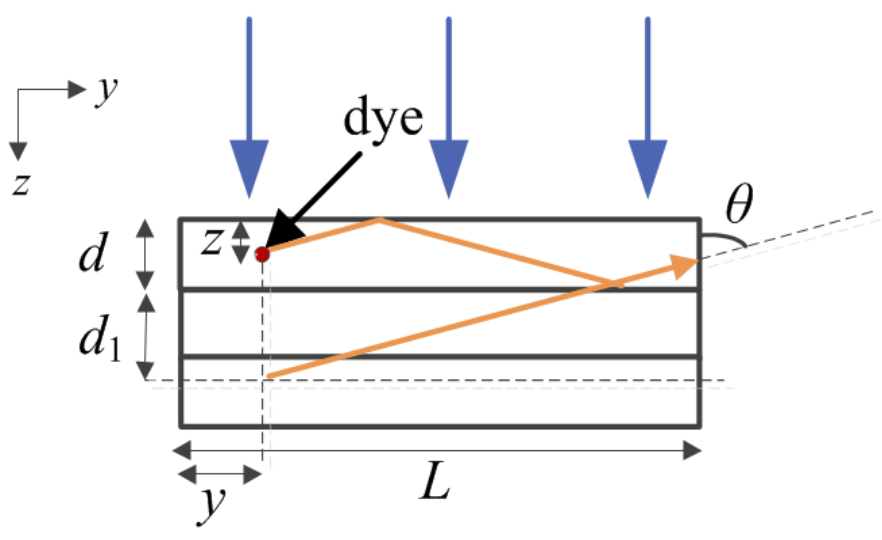

Fig. 1. The top stack is the side view of a LSC. TIR path length is equivalent to straight propagation in a stack of LSCs.

Consider the LSC to be of thickness $d$. If only light passing through the centre of the detector facing edge (i.e. $z=d / 2$ ) and only rays travelling in the plane of rotation of the detector (i.e. within a narrow solid angle i.e. $\phi$ close to $\pi / 2$ ) is detected, the specific depth, $z$, within the 
LSC at which the light 'seen' by the detector originates, depends on a distance $d_{1}$. For light reaching the detector at zenith angles between $0^{\circ}$ to $90^{\circ}$ (Fig. 1), $d_{1}$ is given by:

$$
d_{1}=\frac{(L-y)}{\tan \theta}-\frac{d}{2}
$$

For emission from $90^{\circ}$ to $180^{\circ}, d_{1}$ is similarly obtained:

$$
d_{1}=(L-y) \tan \left(\theta-\frac{\pi}{2}\right)-\frac{d}{2}
$$

Each layer in the stack shown in Fig. 1 represents one instance of total internal reflection. The depth of emission $z$ depends on whether the number of stacks, $k$, is odd or even. If $k$ is odd then:

$$
z=\left(d_{1}+d\right)-(k-1) d
$$

If $k$ is even:

$$
z=\left(d_{1}+d\right)-\left(d_{1}-\{k-1\} d\right) .(6)
$$

The total photon flux escaping a LSC, $\phi_{\text {out, }}$ is a summation over all the different generations of luminescence:

$$
\phi_{\text {out }}(\theta)=\phi_{\text {out }, 1}(\theta)+\phi_{\text {out }, 2}(\theta)+\phi_{\text {out }, 3}(\theta) \ldots(7)
$$

Since $\Gamma_{1}$ depends on $A_{1}$ which in turn is different for each generation of luminescence, each term in the infinite series needs to be calculated individually. However, in the case of uniform absorption across the LSC thickness (i.e. $A_{1}=1$ ), similar to [5][27], it can be shown that in the case of uniform absorption across the LSC thickness, the photon flux reaching the edge is 
directly proportional to the first generation transmission probability i.e. $\phi_{\text {out }}$ is also given by $(1)$.

\section{Materials and methods}

Two molded PMMA based LSCs doped with different concentrations of BASF Lumogen F Red 305 dye (supplied by Teknova AS) were characterized. This dye has been developed to have a high luminescence quantum yield, a broad absorption spectrum and a good photostability [28-29]. Dye concentrations of $300 \mathrm{mg} / 1$ and $800 \mathrm{mg} / \mathrm{l}$ and LSC dimensions equal to $25 \times 25 \times 1 \mathrm{~mm}$ were selected.

The emission was analysed as a function of the angle from the edge of the LSC by attaching a square silicon photo diode (Thorlabs, $1 \mathrm{~mm} \times 1 \mathrm{~mm}$ ) to the edge of a motorised system. The detector was setup to move in a semi-circle at a distance of $5 \mathrm{~cm}$ about the central point of this edge, in a plane perpendicular to the LSC orientation, (Fig. 2 shows the setup and the plane of rotation of the detector). The emission spectrum of the edge emission was also analysed as a function of the emission angle and an iris diaphragm was attached at the edge of the detector. The LSC was illuminated uniformly by a LED Luxeon light source. The excitation wavelength was at $510 \mathrm{~nm}$ (see additional information for LED excitation profile). The set-up and experimental method followed that reported in [23]. 


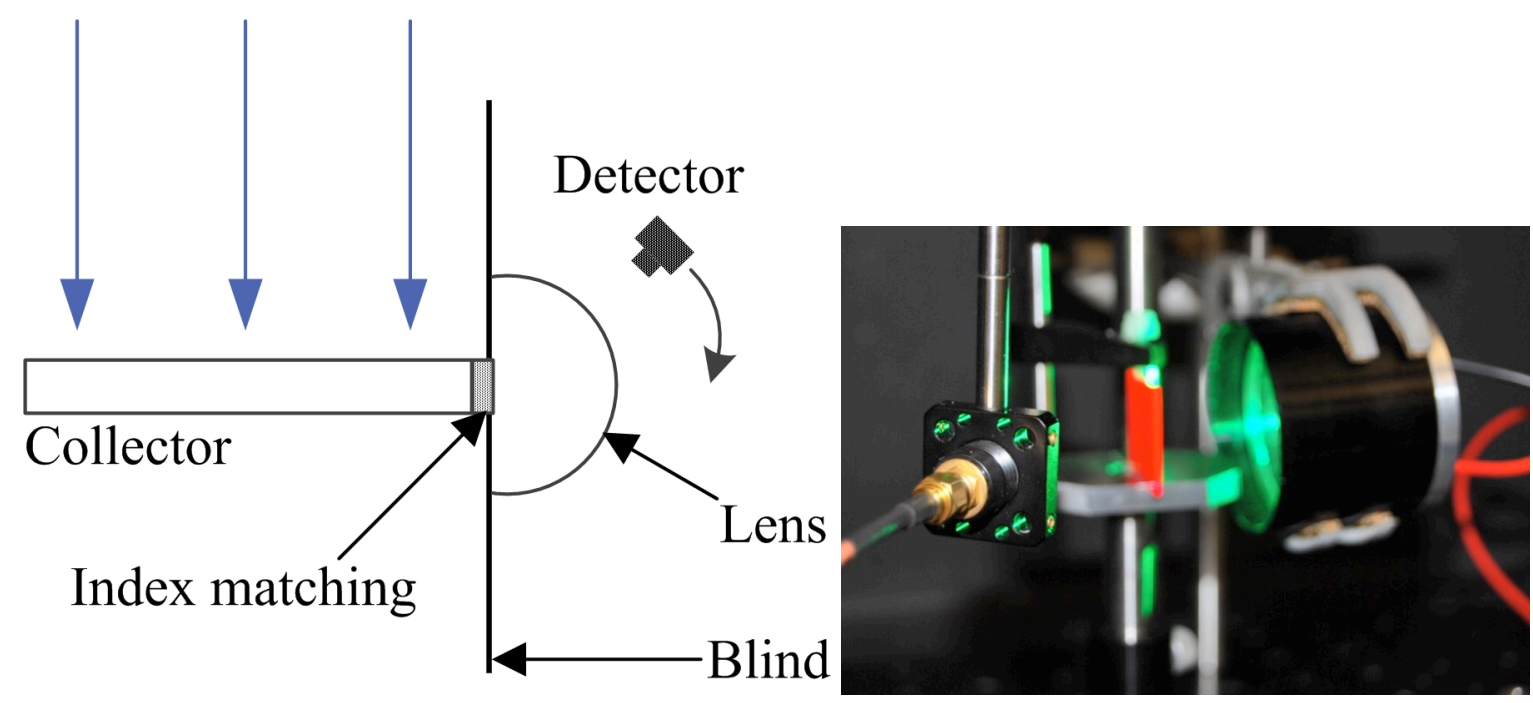

Fig. 2. Experimental set-up for the measurement of the angular distribution escaping the LSC edge (left). The detector is set to move in a semi-circle around the LSC edge, in the plane shown in the left. The detector's 'view' towards the edge is shown (the blind is absent for clarity) (right).

An AvaSpec-2048 spectrometer (Avantes) was used for the measurement of the spectral emission distribution. A single $15 \mathrm{~mm}$ focal length lens was attached at the edge of the LSC with its centre aligned to the LSC edge centre as shown in Fig. 2. The photo diode was also aligned to point at the centre of the LSC edge and the attached iris diaphragm was adjusted to restrict the angular range detected. This ensured that only light with minimal refraction or reflection affecting its path and passing close to the centre line of the LSC edge was detected. An optical coupling medium (Thorlabs) of similar refractive index as the collector $(n=1.5)$ was used to couple the edge of the LSC with the lens. Finally, a thin black surface was used as a blind in order to block any light coming from directions other than the edge of the LSC towards the detector. A photo of the set-up before the attachment of the blind is illustrated in Fig. 2. 
The effect of different directions of illumination was examined by measuring the edge emission under illumination in three configurations: from top surface, bottom surface and illuminating both surfaces simultaneously (Fig. 3). In this manner, the absorption profile of the LSC was altered every time the illumination conditions were changed.

The effect of re-absorption of the luminescence transported in the LSC was studied by measuring the edge emission spectrum of the LSC with a spectrometer attached to a motor. The effect of white scattering layers and silver mirrors attached at bottom surface of the collector on the angular distribution was also examined.

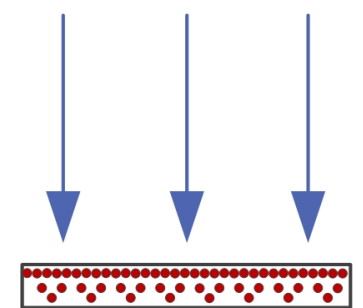

Collector
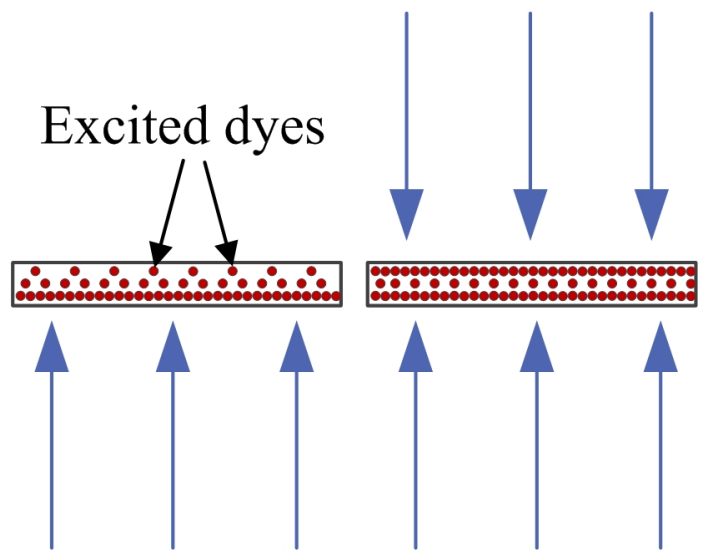

Fig. 3. Schematic illustration of the different positions of the illumination source with respect to the LSC. The sample is illuminated from $0^{\circ}$ (left), $180^{\circ}$ (middle) and both directions (right) changing the absorption profile of the LSC. Depending on the illumination set up the distribution of the 'first generation' sources of emission is shown.

\section{Results and Discussion}

Fig. 4 shows the angular distribution of the luminescence emitted at the edge of a molded sample with high dye concentration $(800 \mathrm{mg} / \mathrm{l})$ when illuminated from only the front side. For convenience of analysis, the distribution is divided into seven areas which represent different characteristics of the distribution. 


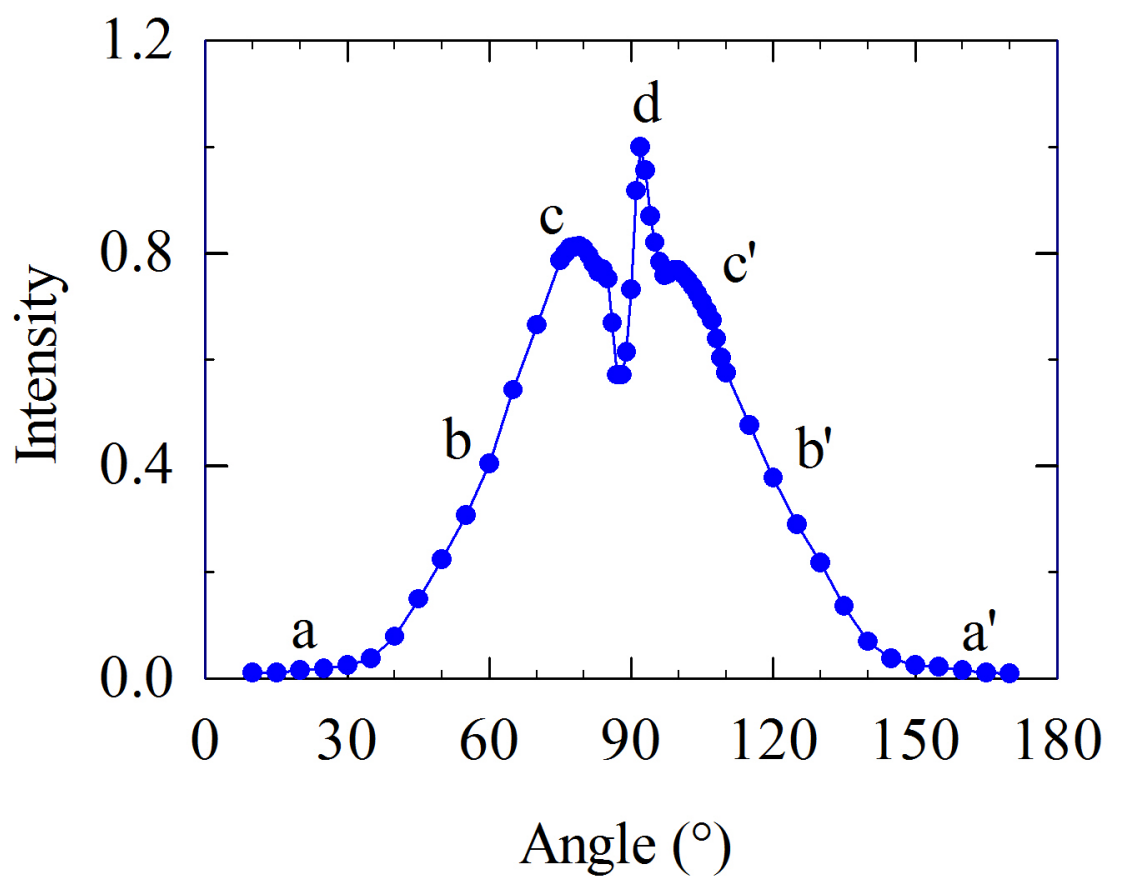

Fig. 4. Angular distribution of the light intensity detected from the edge of a molded high dye concentration $(800 \mathrm{mg} / \mathrm{l})$ LSC. The areas (a-a') correspond to different areas of the distribution.

Fig. 5 shows a typical distribution of molecules excited by the external illumination (i.e. the 'first generation' sources of luminescence) when a LSC is illuminated from the top. Clearly, the non-uniform distribution of excited molecules will be enhanced for high values of the absorbance. This distribution of first generation sources can be used to understand the features of angular emission shown in Fig. 4. Areas a and a' in Fig. 4 are affected principally by the top and bottom escape cones. Below $40^{\circ}$ and above $140^{\circ}$ the intensity is low because most of the light is lost from the top and bottom of the LSC and does not reach the edge. The 
slight intensity measured in these regions is the contribution from molecules emitting close enough to the edge that no reflections occur between emission and the edge.

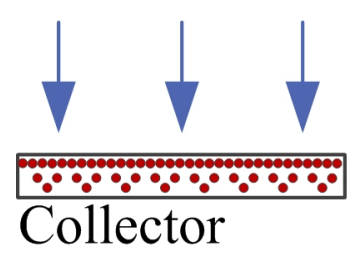

(a)

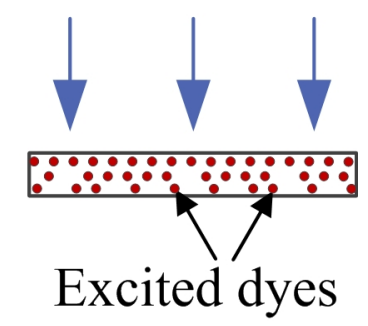

(b)

Fig. 5. Distribution of first generation sources of emission within a (a) high concentration and (b) a low concentration sample.

As the path length decreases (Fig. 6), the intensity of light within the LSC is expected to increase (i.e. areas b and b') due to less re-absorption and non-ideal losses.

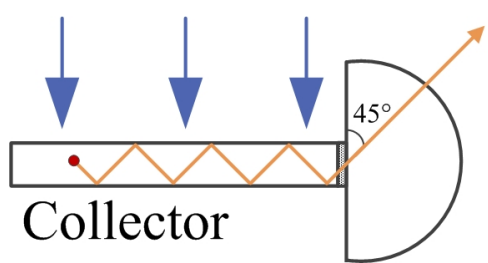

(a)

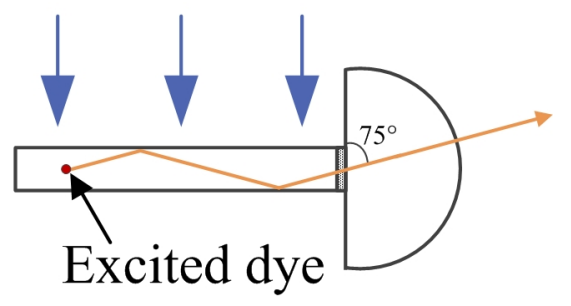

(b) 
Fig. 6. Path length of light that is emitted at (a) $45^{\circ}$ and (b) $75^{\circ}$ with respect to the normal to the LSC top surface. As the angle approaches $90^{\circ}$, the path length of light decreases, leading to an increase in the intensity measured at the edge.

The most interesting characteristic of the angular distribution is the anisotropy observed at around $90^{\circ}$ in areas c-d-c'. This anisotropy is closely related to the point of emission of light within the LSC (Fig. 7). When the detector is at a position slightly below $90^{\circ}$ it 'sees' direct light from sources emitted close to the bottom surface but only light emitted close to the top surface which has been reflected by the bottom surface. The latter requires emission to occur farther away from the edge and therefore corresponds to a longer path length (i.e. higher probability of re-absorption). The opposite applies beyond $90^{\circ}$. Since there are more first generation sources close to the LSC top surface, a large photon flux is detected at angles that 'see' light emitted close to the top surface with lower re-absorption i.e. angles beyond $90^{\circ}$. 


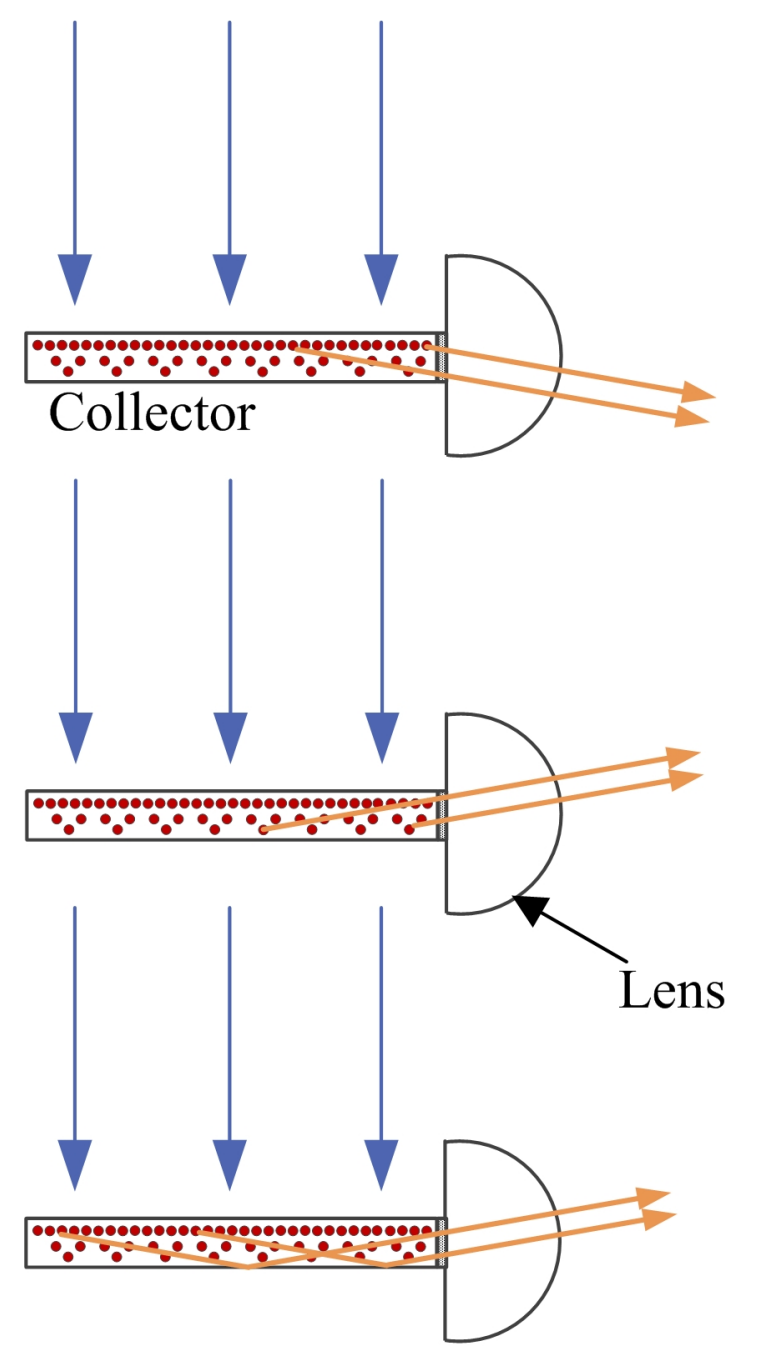

Fig. 7. 'Direct' (top two) and 'reflected' (bottom) emission that exit the edge. At angles below $90^{\circ}$ the detector can see 'direct' light only from emission originating close to the bottom surface (middle). Light originating close to the top surface have to be first reflected by the bottom surface and then reach the detector (bottom). Emission close to the top surface is higher due to the non-uniform absorption across the LSC thickness.

The effect of re-absorption of the luminescence light transport was studied by measuring the edge emission spectrum of the LSC. The critical step in analysing the data from the edge emission of the LSC was the normalization of the edge emission spectrum in the longwavelength luminescence tail as described in [27]. The long wavelength luminescence tail corresponds to a weak absorbance and therefore it is not expected that emission at these 
wavelengths will be distorted by re-absorption effects. Fitting this region to the first generation luminescence allows the effect of re-absorption on emission at shorter wavelengths to be quantified from the relative decrease in emission with respect to the first generation luminescence.

Fig. 8 illustrates the normalised spectrum from the edge emission at $88^{\circ}-92^{\circ}$ for the high dye concentration sample. This corresponds to the area from the trough to the peak in the anisotropic region, as seen in Fig. 4. The red-shift observed on comparing the spectral emission from different angles clearly indicates that re-absorption decreases from $88^{\circ}$ to $92^{\circ}$. As expected, this corresponds to an increase in photon flux from $88^{\circ}$ to $92^{\circ}$. Since the excitation light from the LED source (See Fig. A1 in Additional Information) occurs at a wavelength well below $600 \mathrm{~nm}$ there will not be any effect of the excitation light on the emission results presented in Fig. 8 .

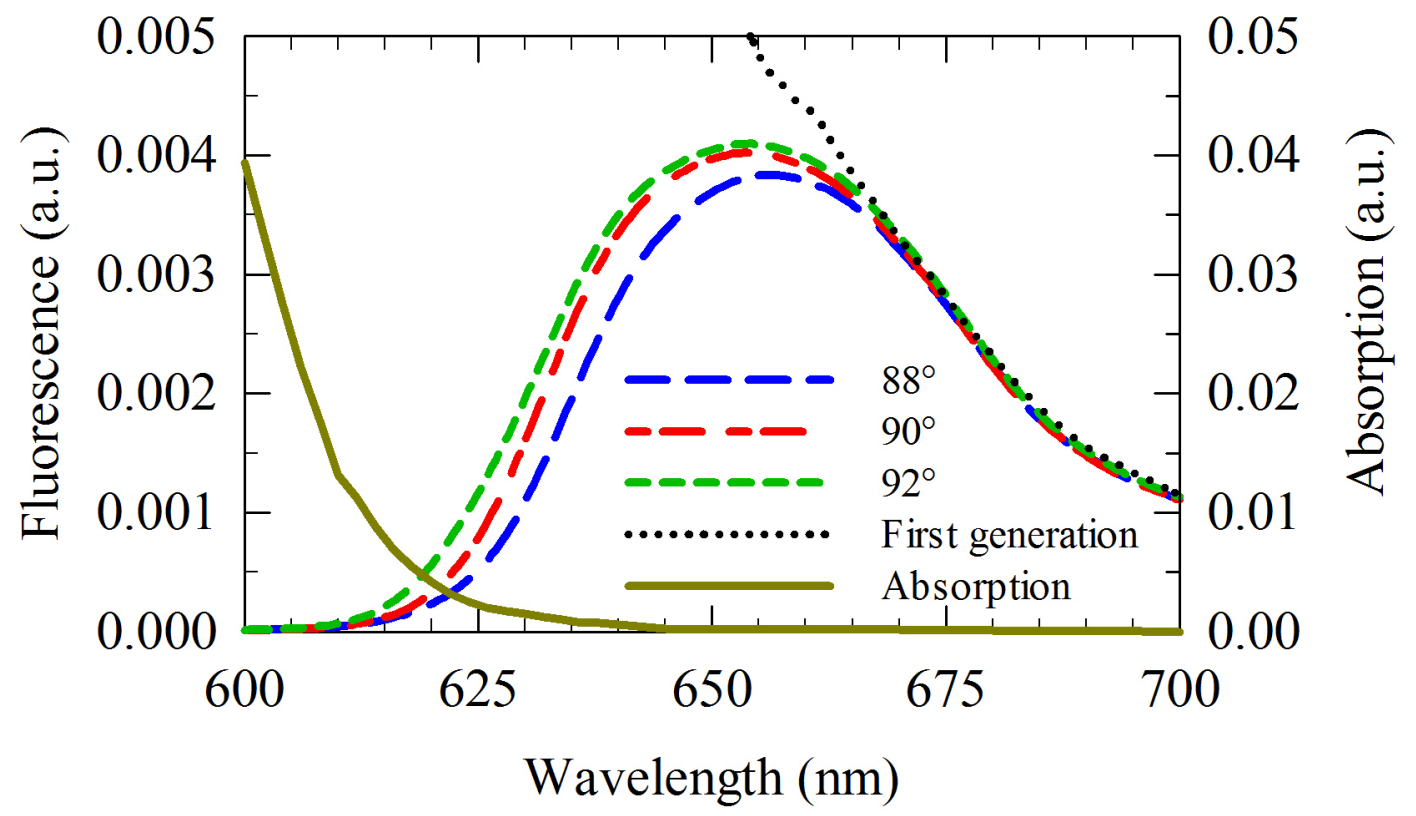

Fig. 8. Normalized edge emission spectra at $88^{\circ}-92^{0-}$ for the high concentration $(800 \mathrm{mg} / \mathrm{l})$ LSC. The tail of the absorption spectrum and 'first generation' emission is shown as well. 
The normalization of the edge emission spectra was carried out according to methods developed in [27].

It is observed that using (1), to describe $\phi_{\text {out }}$, with $\Gamma_{1}$ calculated by selecting an effective $\alpha_{\text {em }}$ and $\alpha_{\mathrm{ex}}$, a key feature of the anisotropy of the experimental results can be obtained. Selecting $\alpha_{\mathrm{em}}$ equal to $0.1 \mathrm{~mm}^{-1}$ and $\alpha_{\mathrm{ex}}$ equal to $1.9 \mathrm{~mm}^{-1}$ the characteristic peaks of the experimental results close to $90^{\circ}$ are reproduced as shown in Fig. 9. Adding an angular loss factor $(\theta)$ equal to $[\sin (\theta)]^{7}$ was observed to match the experimental results at all angles. The dependence of the $(\theta)$ with $\sin (\theta)$ indicates that the loss observed is due to either an increase in the path length or due to a larger number of total internal reflections. The latter could result in losses due to surface imperfections. The exact source of this loss is however unclear from the measurements conducted.

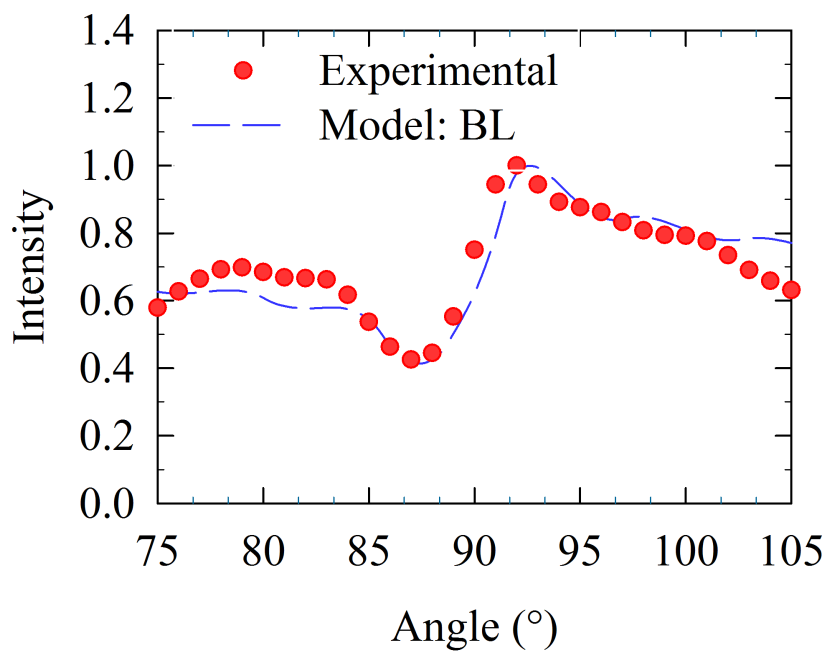

Fig. 9. Angular resolved measurement of the high dye concentration LSC compared to model. In the model $A_{1}$ is assumed to take a Beer-Lambert distribution and an effective $\alpha_{\mathrm{em}}$ and $\alpha_{\text {ex }}$ was selected. The effect of $(\theta)$ was neglected (set to 1 ). 
The effect of LSC dye concentration and the illumination method were investigated to demonstrate the effect of the absorption profile on the angular distribution, (Fig. 10 and Fig. 11 respectively). For both high and low dye concentration LSCs (Fig. 10) the overall shape of the distribution is the same. The difference between the two distributions is the degree of anisotropy about $90^{\circ}$. The variation in the intensity for the low dye concentration sample is not as pronounced as it is for the high dye concentration sample due to weaker absorption and therefore a more uniform absorption profile with thickness.

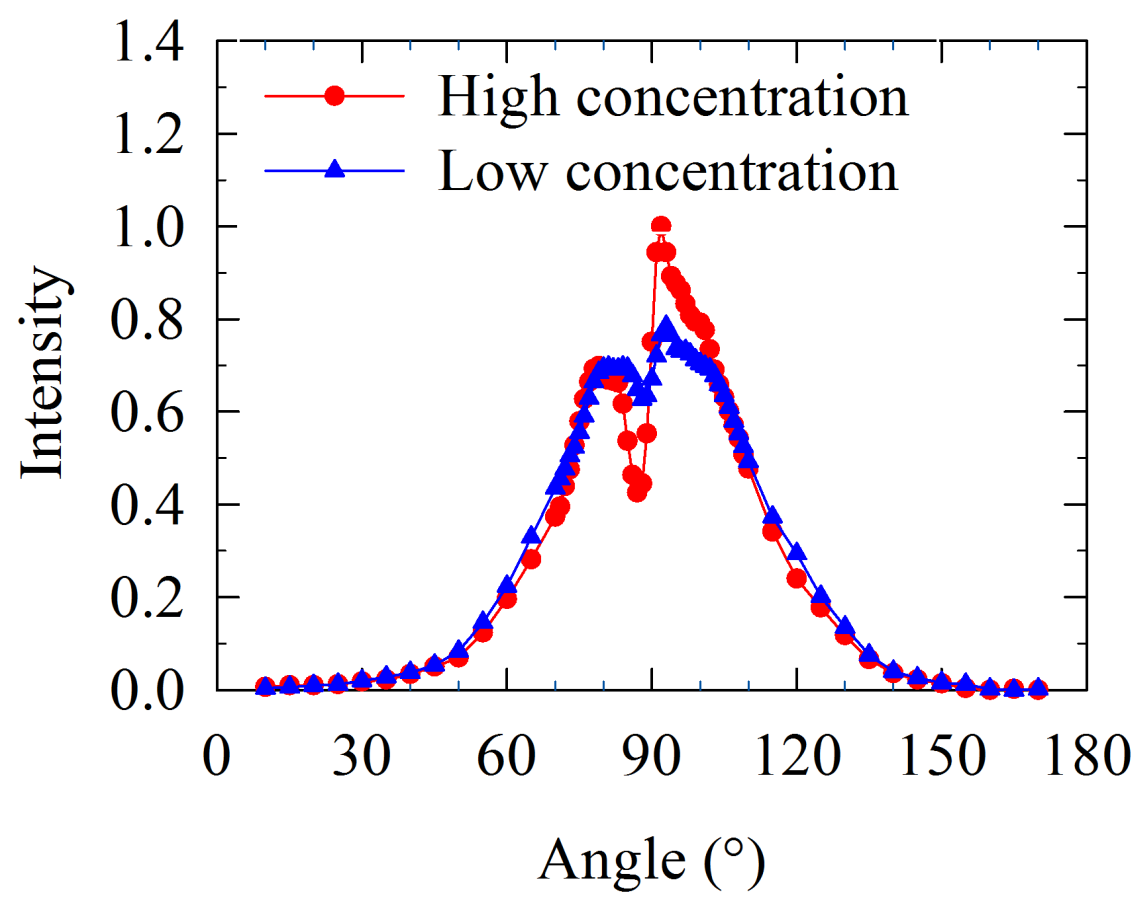

Fig. 10. Comparison of the angular distribution between the high dye concentration $(800$ mg/l) and low dye concentration (300 mg/l) BASF Lumogen F Red 300 LSC samples.

Illumination of the sample by two identical sources simultaneously (at $0^{\circ}$ and $180^{\circ}$ ) yields a symmetrical distribution about $90^{\circ}$ as shown in Fig. 11. Changing the illuminated face is also seen to result in a mirror symmetry of the angular distribution detected. These results are 
again consistent with the anisotropic peaks observed being a result of the absorption profile of the LSC with thickness.

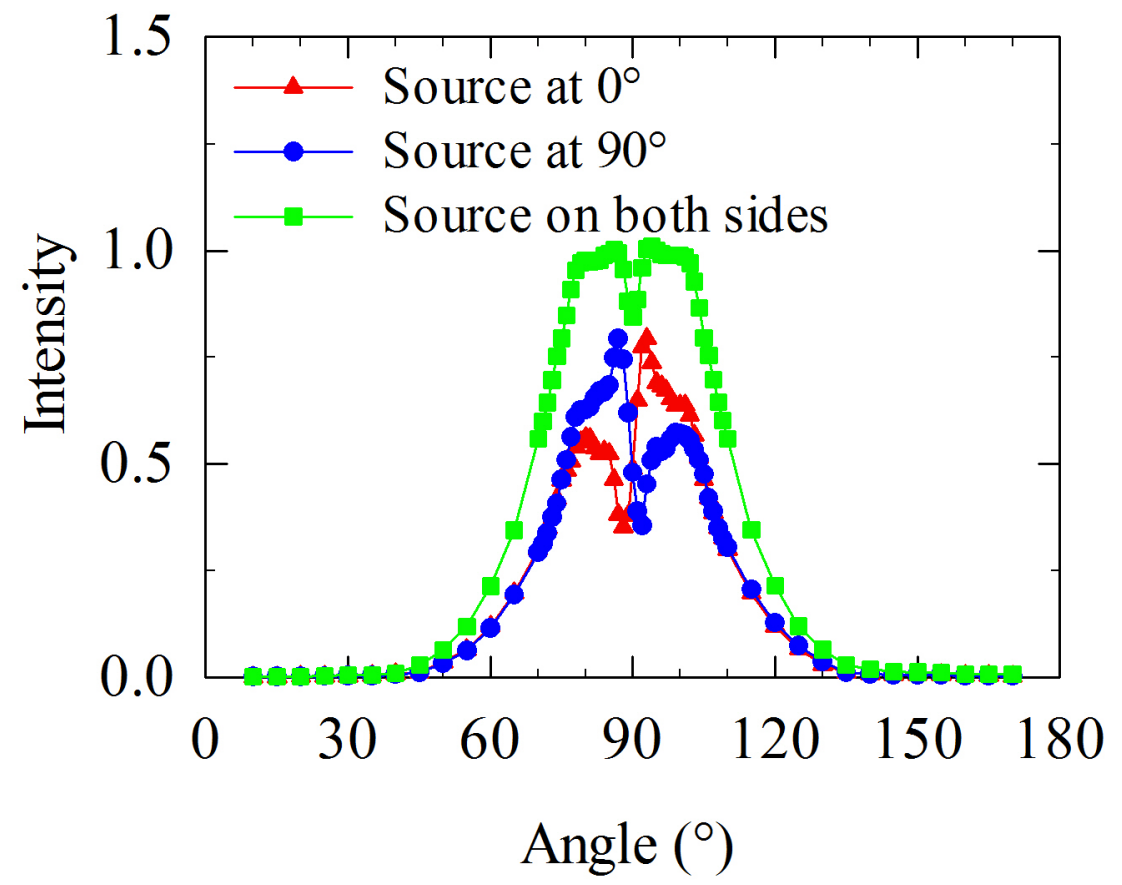

Fig. 11. Angular distribution of emitted light intensity arising from the illumination of the LSC sample $(800 \mathrm{mg} / \mathrm{l})$ with two identical sources, one source positioned at 0 degrees and one source at 180 degrees.

The effect of white scattering layers and silver mirrors attached at the rear surface of the LSC and their effect on the angular distribution was also examined. The results can be seen in Fig. 12.

The results below $90^{\circ}$ indicates that the silver mirror with its specular reflectance, directs rays emitted close to the top surface and into the bottom escape cone to the edge. Without the mirror these rays would be lost through the bottom escape cone. The scattering layer, 
however deflects photons on course for the detector to other angles. This effect is stronger at steep angles, as seen in Fig. 12 due to the corresponding larger number of scattering events. Beyond $90^{\circ}$ the silver mirror and the white layers give result in equal intensities due to the fact that their contribution is mainly from re-directing light back into the collector. The absorbance is effectively increased by giving a second chance for light that would otherwise be lost from the bottom surface. 


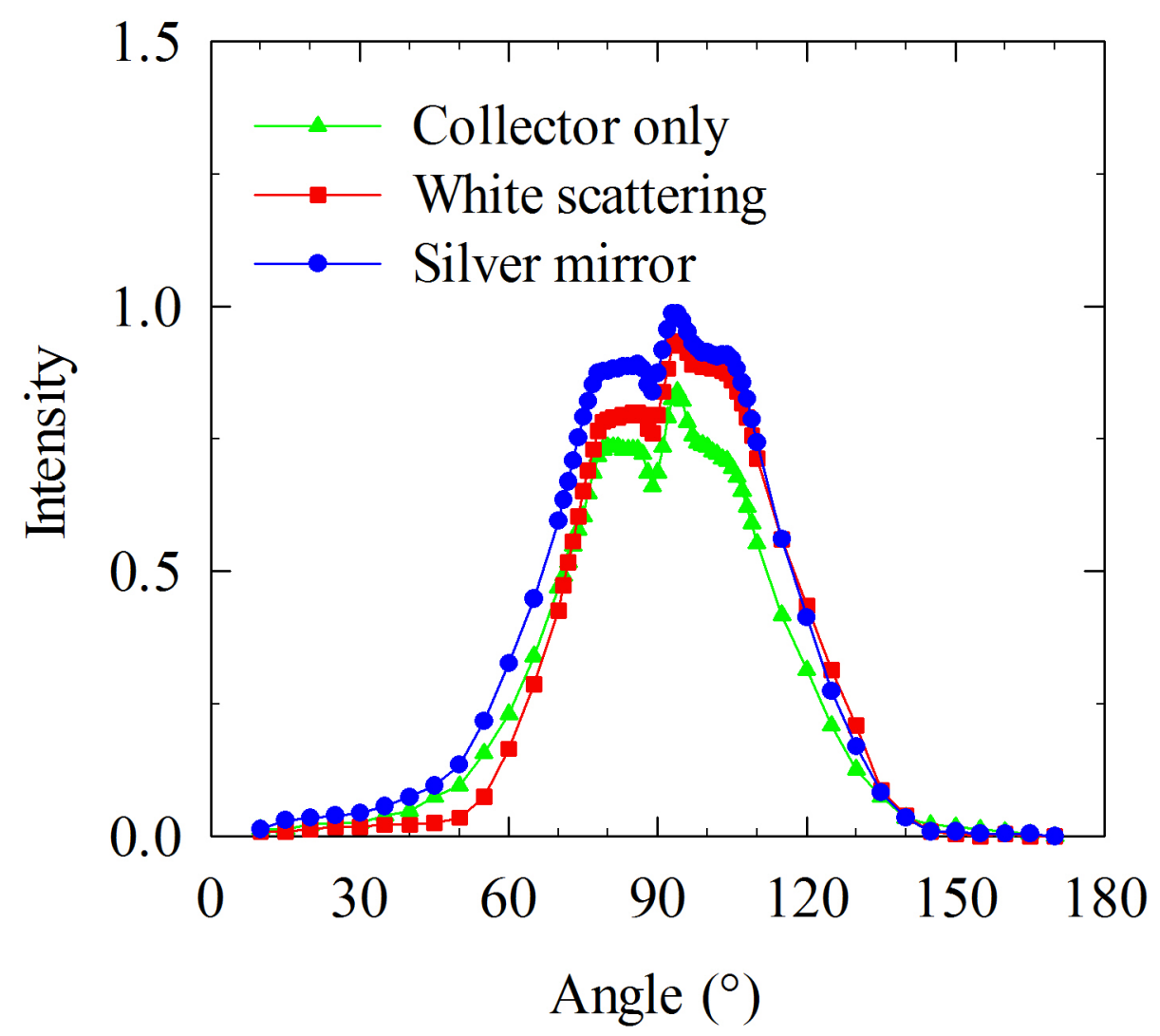

Fig. 12. The effect of the silver mirror and white scattering layer, in the low dye concentration sample. The silver mirror has the greatest positive effect on the distribution while the white scatterer seems to reduce the intensity at certain angles. 


\section{Conclusions}

We have presented a new model describing the angular dependence of the photon flux exiting the LSC edge. It has been shown that the main features of the experimentally measured luminescence emission from the edge of the LSC are consistent with the model developed. The effect of bottom surface reflectors on the photon flux exiting the LSC edge has also been presented for the first time. Our modelling and explanations of the angular distribution of emission exiting LSCs is consistent with the analysis based on different illumination methods, and with spectroscopic results of the emitted light as a function of the emission angle.

\section{Acknowledgements}

The authors would like to gratefully acknowledge Dr Robert Greef for valuable suggestions with the optical set up and characterisation of the samples. Thanks to the financial support from the Faculty of Engineering and the Environment at the University of Southampton and the Engineering and Physical Sciences research council (EPSRC).

\section{References}

[1] M.G. Debije, P.P.C. Verbunt, Thirty years of luminescent solar concentrator research: solar energy for the built environment, Advanced Energy Materials 2 (2012), 12-35.

[2] W.H. Weber, J. Lambe, Luminescent greenhouse collector for solar radiation, Applied Optics 15 (1976), 2299-2300.

[3] A. Goetzberger, W. Greubel, Solar energy conversion with fluorescent collectors, Journal of Applied Physics 14(1977), 123-139. 
[4] M. Currie, J. Mapel, T. Heidel, S. Goffri, M. Baldo, High-efficiency organic solar concentrators for photovoltaics, Science 321(2008), 226-228.

[5] J.S. Batchelder, A.H. Zewail, T. Cole, Luminescent solar concentrators 1: theory of operation and techniques for performance evaluation, Applied Optics 18(1979), 30903110.

[6] J.S. Batchelder, A.H. Zewail, T. Cole, Luminescent solar concentrators 2: experimental and theoretical analysis of their possible efficiencies, Applied Optics 20(1981), 37333754.

[7] L.R. Wilson, B.C. Rowan, N. Robertson, O. Moudam, A.C. Jones, B.S. Richards, Characterization and reduction of re-absorption losses in luminescent solar concentrators, Applied Optics 49(2010), 1651- 1661.

[8] T. Wang, J. Zhang, W. Ma, Y. Luo, L. Wang, Z. Hu, W. Wu, X. Wang, G. Zou, Q. Zhang, Luminescent solar concentrator employing rare earth complex with zero selfabsorption loss, Solar Energy 85 (2011), 2571-2579.

[9] K.W.J. Barnham, J.L. Marques, J. Hassard, P. O’Brien, Quantum dot concentrator and thermodynamic model for the global redshift, Applied Physics Letters 76(2000), 11971199.

[10] A.J. Chatten, K.W.J. Barnham, B.F. Buxton, N.J. Ekins-Daukes, M.A. Malik, A new approach to modeling quantum dot concentrators, Solar Energy Materials and Solar Cells 75(2003), 363-371. 
[11] S.J. Gallagher, B. Norton, P.C. Eames, Quantum dot solar concentrators: electrical conversion efficiencies and comparative concentrating factors of fabricated devices, Solar Energy 81(2007), 813-821.

[12] A.H. Zewail, J.S. Batchelder, Luminescent solar concentrators: an overview, Polymers in Solar Energy Utilisation, 220 (1983), 331-337.

[13] A. Goetzberger, V. Wittwer, Fluorescent planar collectors-concentrators: A review, Solar Cells 4(1981), 3-23.

[14] W. Levitt, and J. Weber, Materials for luminescent greenhouse solar collectors, Applied Optics 1(1977), 2684-2689.

[15] L.H. Slooff, E.E. Bende, A.R. Burgers, T. Budel, M. Pravettoni, R.P. Kenny, E.D. Dunlop, and A. Büchtemann, A luminescent solar concentrator with $7.1 \%$ power conversion efficiency, Physica Status Solidi (RRL)—Rapid Research Letters 2(2008), 257-259.

[16] T. Markvart, Detailed balance method for ideal single-stage fluorescent collectors, Journal of Applied Physics 99(2006), 1-3.

[17] W. Shockley, H. Queisser, Detailed balance limit of efficiency of p-n junction solar cells, Journal of Applied Physics 32(1961), 510-519.

[18] R.W. Olso, R.F. Loring, M.D. Fayer, Luminescent solar concentrators and the reabsorption problem, Applied Optics 20(1981), 2934-2940. 
[19] B.C. Rowan, L.R. Wilson, B.S. Richards, Advanced material concepts for luminescent solar concentrators, IEEE Journal of Selected Topics in Quantum Electronics 14(2008), $1312-1322$.

[20] M.G. Debije, J. Teunissen, M.J. Kastelijn, P. Verbunt, C. Bastiaansen, The effect of a scattering layer on the edge output of a luminescent solar concentrator, Solar Energy Materials and Solar Cells 93(2009), 1345-1350.

[21] J.C. Goldschmidt, M. Peters, A. Bosch, H. Helmers, F. Dimroth, S.W. Glunz, G. Willeke, Increasing the efficiency of fluorescent concentrator systems, Solar Energy Materials and Solar Cells 93(2009), 176-182.

[22] P.S. Friedman, Luminescent solar concentrators, Optical Engineering 20(1981), 887892.

[23] J.C. Goldschmidt, M. Peters, L. Prönneke, L. Steidl, R. Zentel, B. Bläsi, A. Gombert, S. Glunz, G. Willeke and U. Rua, Theoretical and Experimental Analysis of Photonic Structures for Fluorescent Concentrators with Increased Efficiencies, Physica Status Solidi A 205(2008): 2811-2821.

[24] J.C. Goldschmidt, Novel Solar Cell Concepts, Verlay Dr. Hut, München, 2009.

[25] M. Bendig, J. Hanika, M. Peters, H. Dammertz, M. Weber and J.C. Goldschmidt, Simulation of Fluorescent Concentrators, IEEE/EG Symposium on Interactive Ray Tracing 2008, 93-98. 
[26] H. Sträter, S. Knabe, T.J.J. Meyer, G. Bauer, Spectrally and angle-resolved emission of thin film fluorescence collectors, Progress in Photovoltaics: Research and Applications 21(2011), 554-560.

[27] P. Kittidachachan, L. Danos, T.J.J. Meyer, N. Alderman, T. Markvart, Photon collection efficiency of fluorescent solar collectors, Chimia 61(2007), 780-786.

[28] G. Seybold, G. Wagenblast, New perylene and violanthrone dyestuffs for fluorescent collectors, Dyes and Pigments 11 (1989), 303-317.

[29] BASF Lumogen F data sheets, <http://www2.basf.us/additives/pdfs/p3201e.pdf $>$ (last accessed 05.012.2013). 